\title{
An adaptive fuzzy logic quaternion scaled unscented Kalman filtering for inertial navigation system, GPS and magnetometer sensors integration
}

\author{
Wassim Khoder ${ }^{1}$, Bassem Jida ${ }^{2}$ \\ ${ }^{1}$ Faculty of Economics and Business Administration, Lebanese University, Tripoli, Lebanon \\ ${ }^{2}$ Faculty of Letters, Lebanese University, Tripoli, Lebanon \\ Email address: \\ wassim.khoder@hotmail.com (W. Khoder),bassemjida@hotmail.com (B. Jida)
}

\section{To cite this article:}

Wassim Khoder, Bassem Jida. An Adaptive Fuzzy Logic Quaternion Scaled Unscented Kalman Filtering for Inertial Navigation System, GPS and Magnetometer Sensors Integration. Science Journal of Circuits, Systems and Signal Processing. Vol. 3, No. 2, 2014, pp. 5-13.

doi: $10.11648 /$ j.cssp.20140302.11

\begin{abstract}
In this paper, we present a technique based on fuzzy logic to improve the performance of the inertial navigation system integrated with GPS, and magnetometer. The proposed fuzzy technique is primarily used to predict position and velocity measurements during GPS outage signals. As long as the GPS measurements are available, the Q-SUKF of INS/GPS/MAG (MAG: magnetometer) integrated system operates efficiently and provides precise navigation states estimation. Nevertheless, during GPS outage signals, the proposed fuzzy technique is adapted to the Q-SUKF to obtain the (A) (FL) QSUKF (Adaptive Fuzzy Logic Quaternion Scaled Unscented Kalman Filter) in order to correct the degradation of the performance of the algorithm. The adaptive fuzzy logic attributes values to the measurements covariance matrix in order to determine the gain of the filter. It will decrease the measurement noise variance of the Kalman filter and then improves eventually the accuracy of the integrated navigation system states estimation. Finally, an experimental part on the use of the proposed fuzzy technical with the Q-SUKF has been validated. Several GPS outages with duration of 30s have been simulated to study the behavior of the proposed filter. In addition, an initial attitude error of 60 degrees is given in each axis to test the robustness of the filter proposed under large attitude errors. The results of the experimental validation have shown the effectiveness and the significant impact of the (A) (FL) Q-SUKF in the reduction of the drift errors estimation of the position and velocity in case of GPS outages in the tested scenarios.
\end{abstract}

Keywords: Inertial Navigation System, GPS, Magnetometer, Takagi-Sugeno Fuzzy Model, Fuzzy C-Means, Mean Square Error

\section{Introduction}

The low cost inertial navigation systems provide accurate and reliable navigation solutions when they are integrated with aided sensors (GPS, magnetometer) in the filter QSUKF [1]. The INS/GPS/MAG integration can be briefly classified as loosely-coupled, tightly-coupled and deeplycoupled. The choice of the integration approach depends on the type of application and on the operating environment. The large bias drift of inertial sensors prevent the inertial navigation system to operate in standalone mode and even when it is combined with the magnetometer. Therefore, the performance of the integrated system will deteriorate considerably during periods of GPS outages. During the last decade, the Takagi-Sugeno (TS) fuzzy systems have been used to model successfully the non-linear systems and have proved a good representation of dynamic systems [2], [3], [4], [5]. In these approaches, the non linear behavior of a system is represented by a composition of "If-Then" rules, concatenating a set of local linear sub models. In this article, a Takagi-Sugeno fuzzy model is used to estimate the position and velocity measurements to the integrated INS/GPS/MAG system during the various GPS outages. The fuzzy model requires an offline learning phase extracted from a large number of input-output coupled data when GPS signals are available. This phase aims to identify the parameters of the fuzzy model used with the filter Q-SUKF. The Input-Output data cover different dynamics and types of movement (straight and rotation). During the learning phase, the inputs of the fuzzy model are position, velocity and yaw angle 
predicted by the Q-SUKF filter. The outputs of the fuzzy model are the positions and velocity measured by the GPS. At the end of the learning phase, the best estimates of the parameters of the fuzzy model are achieved. Therefore, the model should be able to provide corrections or compensatory measurements of position and velocity during GPS outages in real time process. These measurements allow to maintain the update mode phase of Q-SUKF fiter. In this paper, we describe a new hybridization filter, (A) (FL) Q-SUKF, of these three sensors based on the application of the fuzzy model with the Q-SUKF filter. Next, an experimental part on the (A) (FL) Q-SUKF algorithm has been validated.

\section{Robustness of the Q-SUKF}

The Q-SUKF is a recursive algorithm designed to correct the errors of the inertial navigation system through external measurements provided by aided sensors which are here, the GPS and magnetometer. As long as the GPS measurements are available, the Q-SUKF operates efficiently and provides a precise estimate of the navigation's states. Nevertheless, during the GPS outages, the overall performance of INS/GPS or INS/GPS/MAG systems is significantly degraded due to the rapid accumulation of errors which affect the inertial measurement unit components of the inertial navigation system.

\subsection{Use of Fuzzy Logic as a Criterion of Robustness of the Q-SUKF Filter}

The fuzzy logic is a set of mathematical theories which deals with the representation and manipulation of imperfect knowledge (imprecise, uncertain or incomplete). It does not seek to eliminate them; on the contrary, it will seek to preserve to the maximum. Therefore, its purpose is to supple the representation framework and knowledge's processing, inspiring thus from the human mental processes. The practical applications of fuzzy logic are numerous. Examples include: automatism, robotics, expert systems, decision support, etc. In this paper, the fuzzy logic is defined as a logic which uses the general function of "expert system" in data processing. A fuzzy model, denoted (A) (FL), is proposed to solve the problem of the performance's degradation of the INS/GPS or INS/GPS/MAG system during the GPS outages. When GPS signals are available, this model is extracted offline from a large number of coupled input-output data during a period called learning phase. The inputs of the fuzzy model are, position, velocity, and yaw angles, predicted by the Q-SUKF. The outputs of the fuzzy model are the positions and velocities measured by the GPS, as shown in Figure 1.

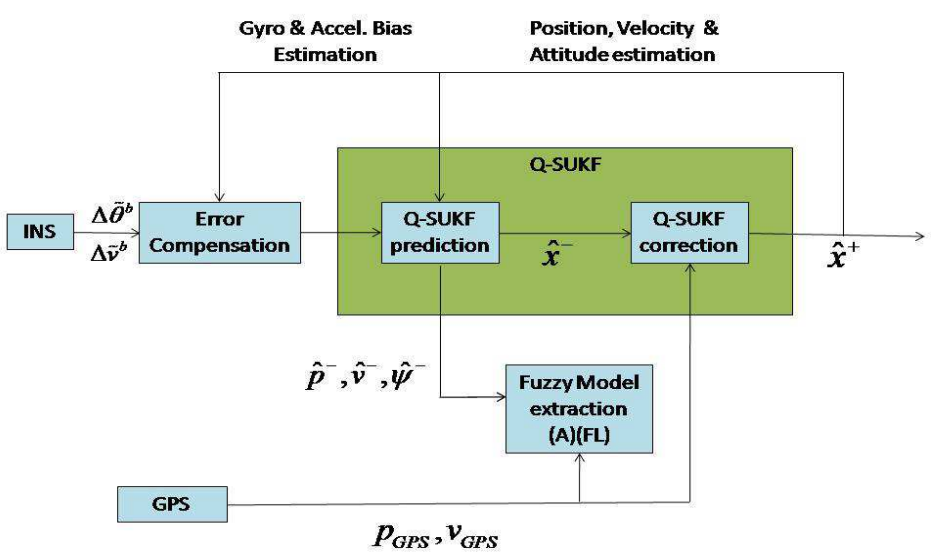

Figure 1. Data collections for the extraction of fuzzy model (A) (FL) during the learning phase.

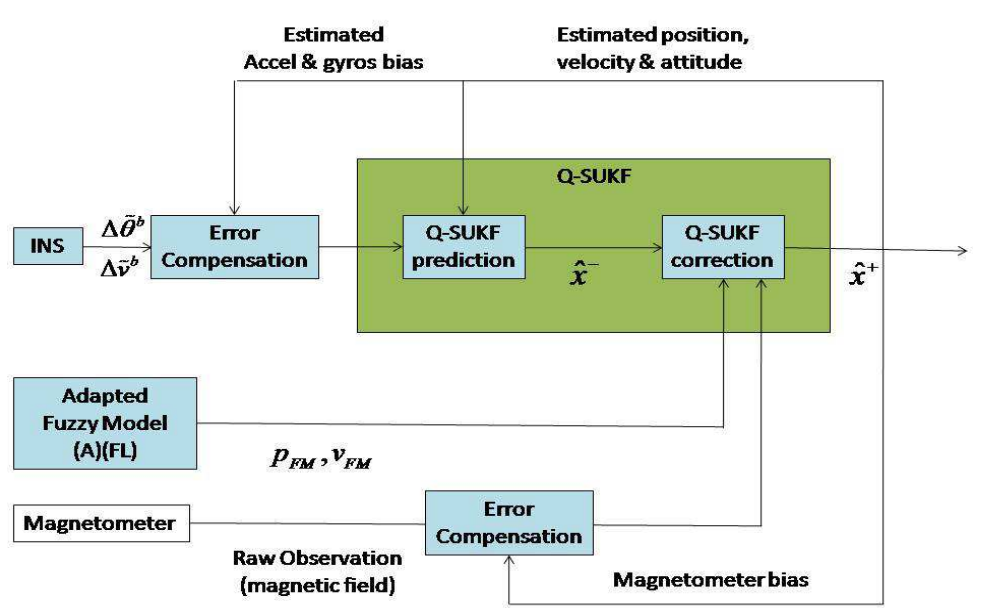

Figure 2. Operating mechanism of the fuzzy model (A) (FL) with the Q-SUKF during GPS outages 
At the end of the learning phase, the best estimates of the parameters of the Fuzzy model are achieved. When an outage GPS occurs, the fuzzy model (A) (FL) generate alternately estimated measurements of position and velocity and which are supposed to be the GPS measurements if they were available. Consequently, the filter Q-SUKF continues to use the equations of measurement update, as shown in Figure 2.

The Q-SUKF filter is noted (A) (FL) Q-SUKF when it is used with the proposed fuzzy model.

\section{Proposed Fuzzy Inference System}

The proposed fuzzy model utilizes a fuzzy inference system of Takagi-Sugeno type (FIS-TS) which has special properties since it represents the non-linear systems in the form of an interpolation between local linear models. The FIS-TS fuzzy model proposed can be written in a general form as:

$$
R_{i} \text { :If } x_{k} \text { is } A_{i}\left(x_{k}\right) \text { Then } \tilde{y}_{i}=a_{i}^{T} x_{k}+b_{i}, i=1, \ldots, r
$$

Where, $A_{i}\left(x_{k}\right)$ is a gaussian membership function of the input variable vector at observation $k, x_{k}$, in the fuzzy set $A_{i}$. $a_{i}$ and $b_{i}$ are the components of the consequent parameters vector $\Theta_{i}=\left[\begin{array}{ll}a_{i}^{T} & b_{i}\end{array}\right]^{T}$ of the $i$-th fuzzy rule which describes the local linear model. $R_{i}$ is the $i$-th fuzzy rule, $r$ is the total number of rules and $\tilde{y}_{i}$ is the estimated output of the local linear model. The proposition " $x_{k}$ is $A_{i}\left(x_{k}\right)$ " can be defined for the different components of $x_{k}$ in form of conjunction:

$$
\begin{aligned}
& R_{i} \text { :If } x_{1, k} \text { is } A_{i, 1}\left(x_{1, k}\right) \text { and } \ldots \text { and } x_{n, k} \text { is } A_{i, n}\left(x_{n, k}\right) \\
& \text { then } \tilde{y}_{i}=a_{i}^{T} x_{k}+b_{i}, i=1, \ldots, r
\end{aligned}
$$

Where $n$ is the number of components of $x_{k}$. The components of $x_{k}$ are the three components of the position $p_{k}^{n}=\left[\begin{array}{lll}\Phi_{k} & \lambda_{k} & h_{k}\end{array}\right]$, the three components of the velocity $v_{k}^{n}$ and the yaw angle $\psi_{k}$. The choice of these parameters as input of the fuzzy model is convenient because they are the main factors to affect the prospected outputs of the fuzzy model (position and velocity vectors). In addition, these states are all determined in the navigation frame and easily obtained from the prediction phase of the Q-SUKF filter. Two classes have been assigned iteratively to each component of the entry vector where a Gaussian function has been implemented to represent the membership degree to each class. Based on a number of entries equal to 7 and the class number equal to 2 , the number of rules is therefore equal to $2^{7}=128$. The estimated outputs of the fuzzy model are the position and velocity vectors, $p^{n}$ and $v^{n}$, expressed in the navigation frame and which can be calculated from the equation (1) of the FIS as follows:

$$
\tilde{y}_{k}=\frac{\sum_{i=1}^{r} \beta_{i}\left(x_{k}\right)\left(a_{i}^{T} x_{k}+b_{i}\right)}{\sum_{i=1}^{r} \beta_{i}\left(x_{k}\right)}
$$

Where $\beta_{i}\left(x_{k}\right)$ denotes the degree of fulfillment of the $i$-th rule:

$$
\beta_{i}\left(x_{k}\right)=\prod_{j=1}^{n} A_{i, j}\left(x_{j, k}\right)=\prod_{j=1}^{n} \exp \left(\frac{-1}{2} \frac{\left(x_{j, k}-V_{i, j}\right)^{2}}{\sigma_{i, j}^{2}}\right)
$$

$V_{i, j}, \sigma_{i, j}^{2}$ represent the center and the variance of the Gaussian fuzzy membership functions respectively. To identify the FIS of the fuzzy model, the antecedent parameters, $V_{i, j}, \sigma_{i, j}^{2}$ and the consequent parameters, $\Theta_{i}$, must be determined.

\subsection{Determination of Antecedent Parameters}

Abonyi in [6] has proposed the Fuzzy C-Means classification algorithm (FCM) to identify the antecedent parameters of Takagi-Sugeno fuzzy model. The FCM algorithm aims to divide the data points into homogeneous classes or groups. Thus, the points in the same class are as similar as possible while points in different classes are as dissimilar as possible. The FCM algorithm, which issued from the works of [7] and improved later by [8], constitutes an important reference among the differents methods of fuzzy coalescence [9] based on the minimization of the objective function, of the form:

$$
J_{F C M}(X, U, V)=\sum_{i=1}^{c} \sum_{k=1}^{N} \mu_{i k}\left\|x_{k}-V_{i}\right\|^{2}=\sum_{i=1}^{c} \sum_{k=1}^{N} \mu_{i k} . D_{i k}^{2}
$$

Where $X$ is the data matrix, $N$ is the number of observations, $\mu_{i}$ is the Fuzzy partition of fuzzy subsets $i, U$ is fuzzy partition matrix (of dimension $c \times N$ ), $V=\left[V_{1}, V_{2}, \ldots, V_{c}\right]$ is a matrix of cluster centroid vectors which must be determined, with $V_{i} \in \mathbb{R}^{n}, 1 \leq i \leq c$, in our case, the number of cluster $c$ is equal to the number of rules $r$ and $D_{i k}=\left\|x_{k}-V_{i}\right\|$ is the Euclidean distance between the observation $x_{k}$ and the Cluster centroid vector $V_{i}$. In the equation (eq.5), the dissimilarity measure expressed by the term $J_{F C M}(X, U, V)$ is the sum of the squares of the distances between each observation $x_{k}$ and the corresponding center $V_{i}$. The effect of this distance is weighted by the degree of activation of the class, $\left(\mu_{i k}\right)^{m}$ corresponding to $x_{k}$. The value of the objective function $J_{F C M}(X, U, V)$ can be seen as a measure of the total variance of $x_{k}$ with respect to the 
centers $V_{i}$. The minimization of the objective function $J_{F C M}(X, U, V)$ is a non-linear optimization problem that can be solved by different methods; the most used is the Fuzzy C-Means (FCM) algorithm [8]. The minimization of the objective function $J_{F C M}(X, U, V)$ can be achieved by finding the cluster centroid vectors $V_{i}$ and the standard deviation $\sigma_{i, j}$ of the membership Gaussian functions iteratively [10]:

$V_{i}^{l}=\frac{\sum_{k=1}^{N} \mu_{i k}^{l-1} x_{k}}{\sum_{k=1}^{N} \mu_{i k}^{l-1}}, \sigma_{i, j}^{2(l)}=\frac{\sum_{k=1}^{N} \mu_{i k}^{l-1}\left(x_{j, k}-V_{i, j}^{l}\right)^{2}}{\sum_{k=1}^{N} \mu_{i k}^{l-1}} 1 \leq i \leq c, 1 \leq j \leq n$

Where the membership degree $\mu_{i k}$ are calculated as follows:

$$
\mu_{i k}=\frac{1}{\sum_{j=1}^{n}\left(D_{i k} / D_{j k}\right)^{2 /(m-1)}}, 1 \leq i \leq c \text { with } \mu_{i k} \in\langle 0,1\rangle \text { and } \sum_{i=1}^{c} \mu_{i k}=1
$$

$m \in[1,+\infty)$ is the fuzziness parameter of the partition. The parameter $m$ influences the form of the classes in the data space of the system. When $m$ approaches the value 1 , the shape of the membership function of each class is close to be
Boolean function $(m \in\{0,1\})$. The partition can range from a hard partition $(m=1)$ to a completely fuzzy partition $(m \rightarrow+\infty)$ where the average of the centers are all equal to the average of $X$. These properties are independent of the optimization method selected [11]. Although the choice of $\mathrm{m}$ depends on the data [12], usually this parameter is initialized to a value between 1,5 and 2,5. The iterative process stops when the partition becomes stable, i.e., when there is no changes significantly of the fuzzy partition matrix between two successive iterations. This is expressed in a general terms by the verification of the expression (8) where the left-hand side reflects a matrix norm and the coefficient $\mathcal{E}$ defines the threshold of convergence:

$$
\left\|U^{(l)}-U^{(l-1)}\right\|<\varepsilon
$$

The expression $U^{(l)}$ represents the fuzzy partition matrix of the $l$ - iteration.

\subsection{Determination of Consequent Parameters}

After the learning of the antecedent parameters using equations (6) and (7), the equation (eq.3) can be rewritten as follows:

$$
\tilde{y}_{k}=\sum_{i=1}^{c} \bar{\beta}_{i}\left(x_{k}\right) \cdot x_{k}^{e} \Theta_{i}=\left[\bar{\beta}_{1}\left(x_{k}\right) \cdot x_{k}^{e} \cdots \bar{\beta}_{c}\left(x_{k}\right) \cdot x_{k}^{e}\right]\left[\Theta_{1} \cdots \Theta_{c}\right]^{T}=\left[\bar{\beta}_{1}\left(x_{k}\right) \cdot x_{k}^{e} \cdots \bar{\beta}_{c}\left(x_{k}\right) \cdot x_{k}^{e}\right] \Theta
$$

Where $x_{k}^{e}=\left[\begin{array}{ll}x_{k} & 1\end{array}\right]$ is the input vector to the fuzzy model augmented by the unit element. $\Theta$ is the $M$-dimensional consequent parameters vector where $M=c \times(n+1) \cdot \bar{\beta}_{i}$ is defined by the following formula:

$$
\bar{\beta}_{i}\left(x_{k}\right)=\frac{\beta_{i}\left(x_{k}\right)}{\sum_{i=1}^{c} \beta_{i}\left(x_{k}\right)}
$$

The linear equation (eq.9) of the consequent parameters vector can be written as follows:

$$
Z \Theta=Y
$$

Given $M$ the number of linear consequent parameters and $N$ the number of learning input-output data, the dimensions of the matrices $Z, \Theta$ and $Y$ are $N \times M, M \times 1$ and $N \times 1$ respectively. As $N$ is always greater than $M(N>M)$, the system of linear equations (eq.9) is an underdetermined system, therefore generally there is no exact or unique solution which can be reached. To get there, the least squares estimation (LSE) method is exploited to minimize the squared distance between the vector $Y$ and the linear combination $Z \Theta$. It is a classical problem that forms the basic in many applications such as linear regression, adaptive filtering and signal processing. The famous formula for solving systems of underdetermined equations uses the pseudo-inverse matrix of $\Theta$ as follows [13]:

$$
\Theta^{*}=\left(Z^{T} Z\right)^{-1} Z^{T} Y
$$

Where $Z^{T}$ is the transpose of $Z \cdot\left(Z^{T} Z\right)^{-1} Z^{T}$ is the pseudoinverse matrix of $Z$ if $\left(Z^{T} Z\right)$ is non-singular. Despite that the equation (eq.12) is expressed in few words, it is very costly in terms of computation time when it comes to the calculation of the inverse of a matrix $Z^{T} Z$ and, in addition, it becomes poorly defined if this matrix is singular. To avoid the large computation time or the problem of singularity, sequential formulas are used to calculate the least square estimation of $\Theta$. This sequential method is more efficient, especially when $M$ is small. If the $i^{\text {th }}$ row of the matrix $Z$ in equation (eq.11) is denoted by $z_{i}^{T}$ and the $\mathrm{i}^{\text {th }}$ element of the vector $\mathrm{Y}$ is denoted by $y_{i}^{T}$, then $\Theta$ may be calculated iteratively using the following sequential formulas [13], [14]:

$$
S_{i+1}=S_{i}+\frac{S_{i} z_{i+1} z_{i+1}^{T} S_{i}}{1+z_{i+1}^{T} S_{i} z_{i+1}}, \Theta_{i+1}=\Theta_{i}+S_{i+1} z_{i+1}\left(y_{i+1}-z_{i+1}^{T} \Theta_{i}\right) \quad i=0, \ldots, N-1
$$


Where $S_{i}$ is often called the covariance matrix and the estimated least squares $\Theta^{*}$ is equal to $\Theta_{N}$. The initial condition of the equation (eq.13) is $\Theta_{0}=0$ and $S_{0}=\eta I$, where $\eta$ is an arbitrary positive number which is large and $I$ is the $M \times M-$ dimensional identity matrix.

\section{Simulations}

In this section, the solution of the Q-SUKF filter used with the fuzzy model is presented. The main objective of the (A) (FL) Q-SUKF filter is to preserve the update phase of the QSUKF filter by providing simulated measurements of position and velocity during the GPS outages. The Fuzzy model is extracted from a large number of input/output data through an offline learning phase. This learning phase is realized during the availability or reliability of GPS measurements. The inputs of the fuzzy system are the position, velocity and the yaw angle determined by the QSUKF filter. The outputs of the fuzzy model are the positions and velocities provided by the GPS. The learning phase of fuzzy model is carried out over a short period of time between two successive GPS measurements. This ensures that the drift time of position and velocity errors remain insignificant. It also ensures the reliability of the measurements provided by the learning process. The adaptation of antecedent and consequent parameters of the fuzzy inference system during the learning process uses Fuzzy C-Means (FCM) classification and Least Squares Error (LSE) methods respectively. When GPS outage occurs, the adapted fuzzy logic model (A) (FL) switches to the prediction mode to provide simulated measurements of position and velocity to keep the correction phase in QSUKF filter. The problem now is how to attribute the values of the measurements provided by the proposed fuzzy model to the covariance matrix in order to determine the gain of the filter. To overcome this problem, once the identification parameters of the fuzzy model are calculated, the estimated outputs of the fuzzy model during the learning phase are computed by applying the equation (eq.3). Note that the estimated outputs during the learning phase include the effect of the measurements noises of GPS because they are calculated from the parameters of the fuzzy model identified from these measurements. The variance of these estimated outputs are the diagonals elements of the covariance matrix which must be used as the error covariance matrix of measurements of the fuzzy model during the GPS outages.

\subsection{Simulation Model}

To test the effectiveness of the (A) (FL)Q-SUKF filter and its impact on the accuracy of the navigation parameters calculation (specially the position and velocity), a simulated data of inertial measurement unit, GPS and magnetometer were used. The experiment was conducted using a car driving (reference trajectory) for 30 mimutes. This reference trajectory was generated by the function "progencar" of INS toolbox version 3.0 created by GPSoft. This trajectory covers different dynamic (static and kinematic) and scenarios of motion (rotations and rectilinear). The data of the inertial navigation system (angle and velocity increments) were simulated from the parameters of the profile of the automobile using certain functions of the INS toolbox. These angle and velocity increments have been corrupted with various sources of errors such as biases, scale factors and noises in order to generate outputs close to real data of an inertial navigation system. The characteristics of the error's models of the inertial sensors used in the experiment are presented in Table 1, where the two parameters $T$ and $\sigma$ describe the first-order markov process $x$ represented by:

$$
\dot{x}=-\frac{1}{T} x+\omega
$$

Where $T$ is the correlation time of the process $x$ and $\omega$ is a wiener process with variance $2 \sigma^{2} / T$

Table 1. Characteristics of error models of the inertial sensors used in the experience

\begin{tabular}{llcl}
\hline Parameter & Model & Accelerometer & Gyroscope \\
\hline Noise & Random Walk & $0.6 \mathrm{~m} / \mathrm{s} / \sqrt{h}$ & $3.5 \mathrm{deg} / \sqrt{h}$ \\
& & $\sigma_{a}=0.1 \mathrm{~m} / \mathrm{s}^{2}$ & $\sigma_{g}=100 \mathrm{deg} / \mathrm{h}$ \\
Bias & First-order Gauss-Markov & $T=1 \mathrm{~h} \mathrm{o} \mathrm{u} \mathrm{r}$ & $T=1 \mathrm{hour}$ \\
& & $\sigma_{s a}=1000 \mathrm{PPM}$ & $\sigma_{s g}=1000 P P M$ \\
Scale Factor & First-orderGauss-Markov & $T=4$ hours & $T=4$ hours \\
\hline
\end{tabular}

The GPS data (position and velocity) were generated by adding to the positions and velocities data of the reference trajectory a gaussian white noise. The initial standard deviation of the position expressed in Cartesian coordinates in the navigation frame is equal to $2 \mathrm{~cm}$ in the horizontal plane and is equal to $4 \mathrm{~cm}$ in the vertical plane. The initial standard deviation of the velocity expressed in the navigation frame is equal to $0.25 \mathrm{~m} / \mathrm{s}$ for the horizontal components and is equal to $0.4 \mathrm{~m} / \mathrm{s}$ for the vertical component. Simulated data of the Magnetometer (Earth' s magnetic field) were generated in the navigation frame by the World Magnetic Model 2010 (WMM-2010) that uses the geographic position of the vehicle to determine its components. This reference magnetic field is transformed into the body frame and corrupted by a Gaussian white noise of zero-mean and standard deviation equal to 0,002 Gauss/s 1/2. Two simulations of GPS outages with a duration of $30 \mathrm{~s}$ have been considered along the path, as shown in Figure 3. 
These two GPS outages have been simulated to study the behavior of the (A) (FL) QSUKF filter in the case of degradation of the accuracy of GPS signals. Different dynamics and types of movement (straight and rotation) have been considered during these outages. The vehicule is turned 90 degree to the left when the first GPS outage occurs while the vehicle movement over the second outage of GPS was straight. In these 2 periods, the Q-SUKF filter operates in prediction mode and thus the position and velocity errors should increase with time. On the other hand, the proposed method allows to our filter to operate in update mode where the measurements of correction are obtained from the fuzzy model ((A) (FL) Q-SUKF).

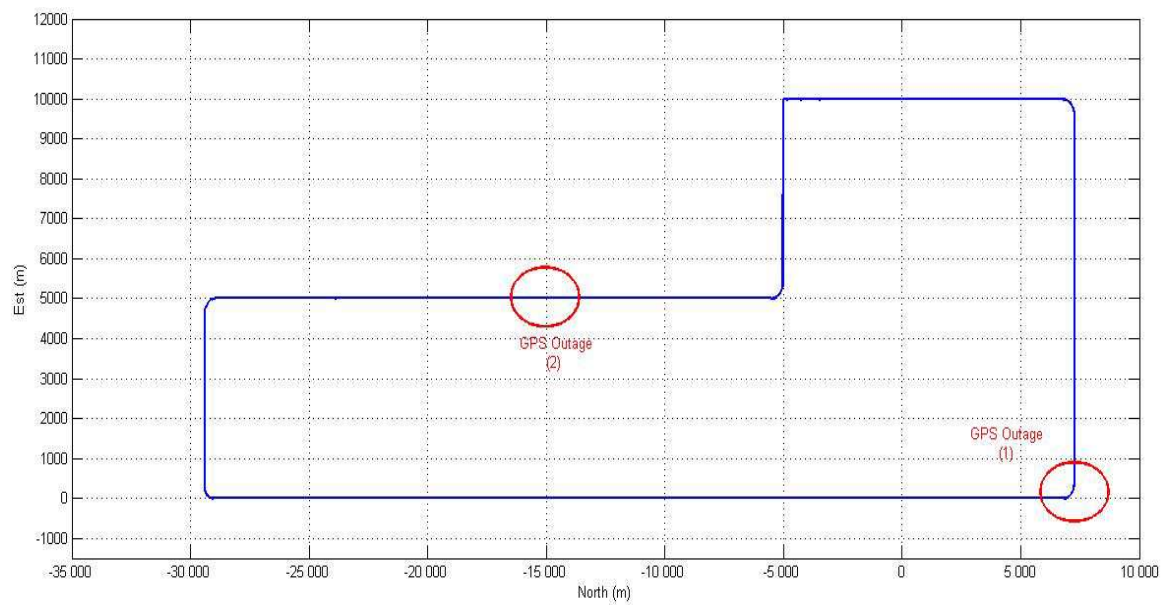

Figure 3. Simulated trajectory with GPS outages indicated.

\subsection{Results}

To test the performance of the proposed approach, an initial attitude error of 60 degrees is given in each axis. The diagonal terms of initial covariance matrix represent variances or mean squared errors. The off-diagonal terms are set to be zeros. The parameters used in the Q-SUKF developed are given by scaling parameters $\alpha=0.05$ and $\beta=2$, and by weight of 0 th point $\omega_{0}=0.5$.

The figures (Figure $4 \mathrm{a}$, Figure $4 \mathrm{~b}$, Figure 5a, Figure $5 \mathrm{~b}$ ) show the velocity errors during the two periods of GPS outages 1, 2 respectively, before and after the application of the proposed technique of the fuzzy model to Q-SUKF filter. We observed in these figures that the maximum errors of the velocity components $\left(\delta v_{n}, \delta v_{e}\right.$ et $\left.\delta v_{d}\right)$ have been reduced after the application of the proposed two periods of GPS outages. The table (Table 2) summarizes the maximum error and the percentage of the reduction of this maximum error for the three components of the velocity. The fuzzy model proposed applied to the Q-SUKF filter has provides a significant improvement of $43 \%$ at least in the reduction of the maximum errors on the different components of the velocity.

Table 2. Reduction of the maximum error of the velocity of Q-SUKF filter after using the fuzzy model.

\begin{tabular}{lll}
\hline Maximum Error & Outage GPS 1 & Outage GPS 2 \\
\hline$\delta \mathrm{v}_{\mathrm{n}} \mathrm{Q}-\mathrm{SUKF}$ & 7,70 & 8,30 \\
$\delta \mathrm{v}_{\mathrm{n}}$ (A)(FL) Q-SUKF & 1,93 & $-1,25$ \\
Reduction (\%) & $75,32 \%$ & $84,93 \%$ \\
$\delta \mathrm{v}_{\mathrm{e}}$ Q-SUKF & & \\
$\delta \mathrm{v}_{\mathrm{e}}$ (A)(FL)Q-SUKF & $-8,20$ & 6,10 \\
Reduction (\%) & 4,60 & 2,10 \\
$\delta \mathrm{v}_{\mathrm{d}}$ Q-SUKF & $43,90 \%$ & $65,57 \%$ \\
$\delta \mathrm{v}_{\mathrm{d}}(\mathrm{A})(\mathrm{FL}) \mathrm{Q}-\mathrm{SUKF}$ & $-2,00$ & $-3,20$ \\
Reduction (\%) & -0.50 & 0,40 \\
\hline
\end{tabular}

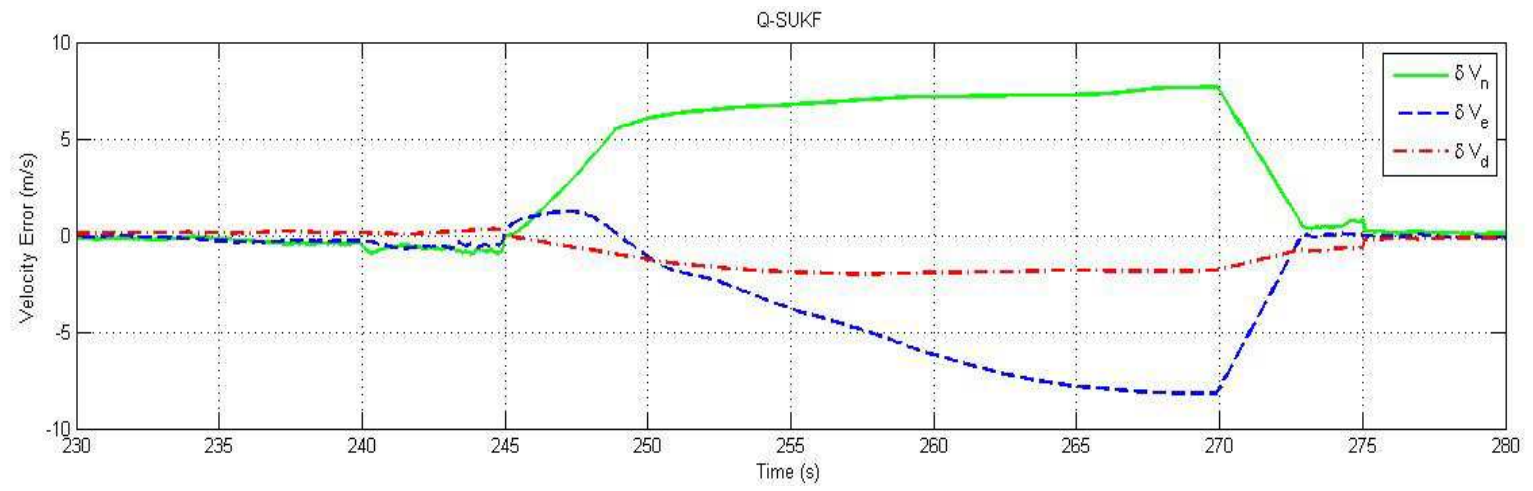

Figure 4a. Velocity error estimated by Q-SUKF during GPS outage 1 


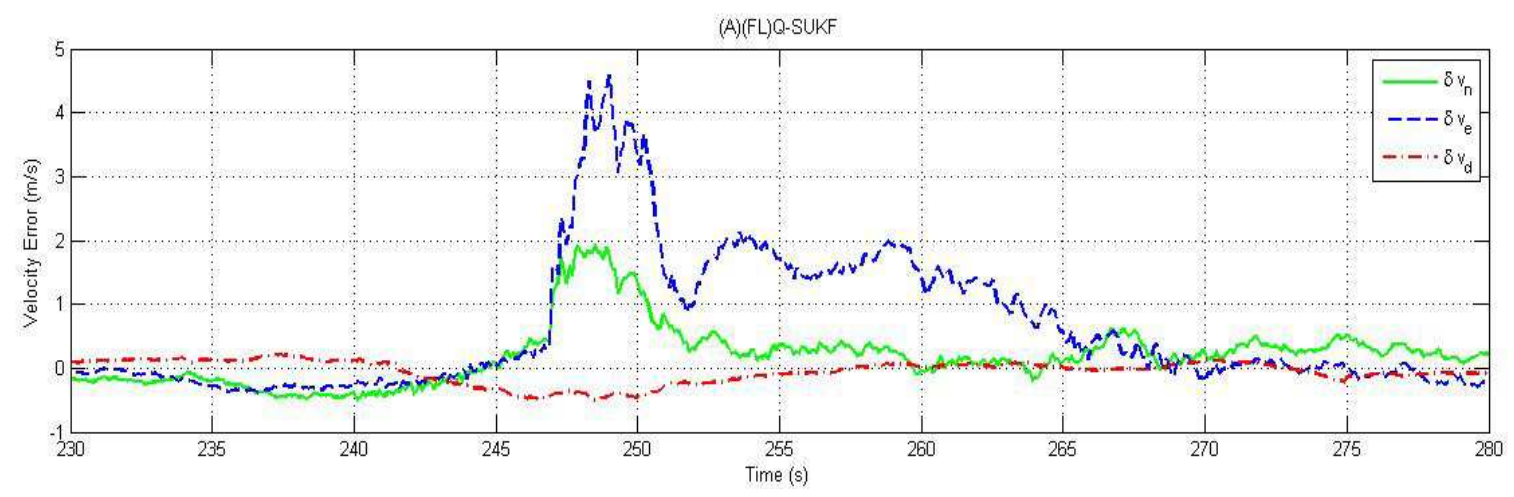

Figure $4 b$. Velocity error estimated by (A)(FL)Q-SUKF during GPS outage 1

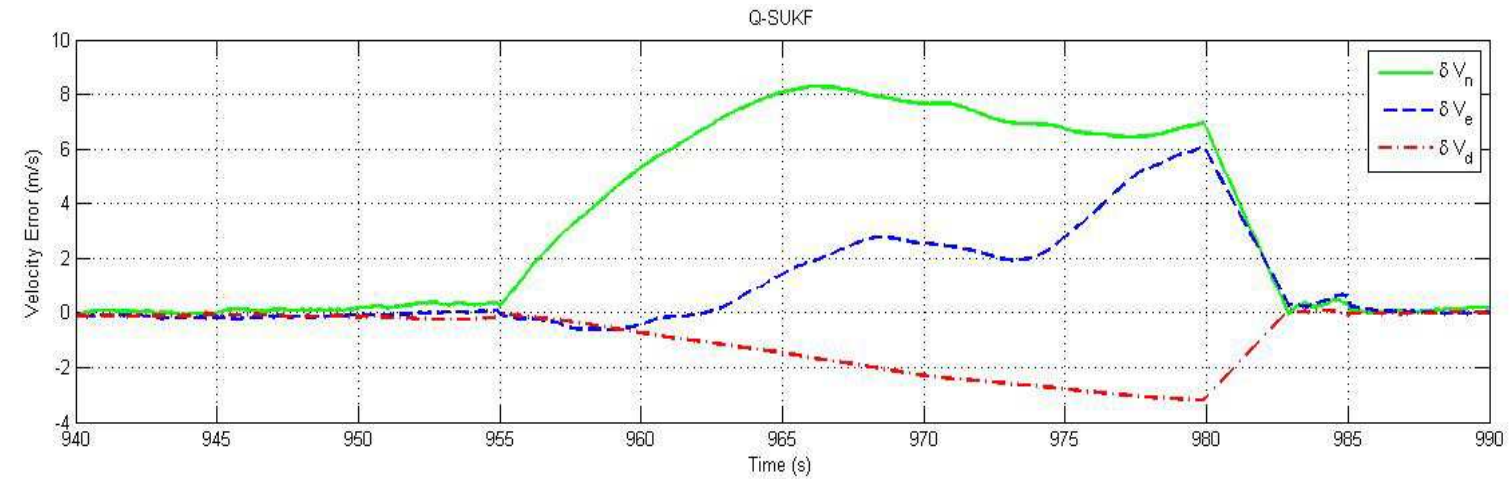

Figure 5a. Velocity error estimated by Q-SUKF during GPS outage 2

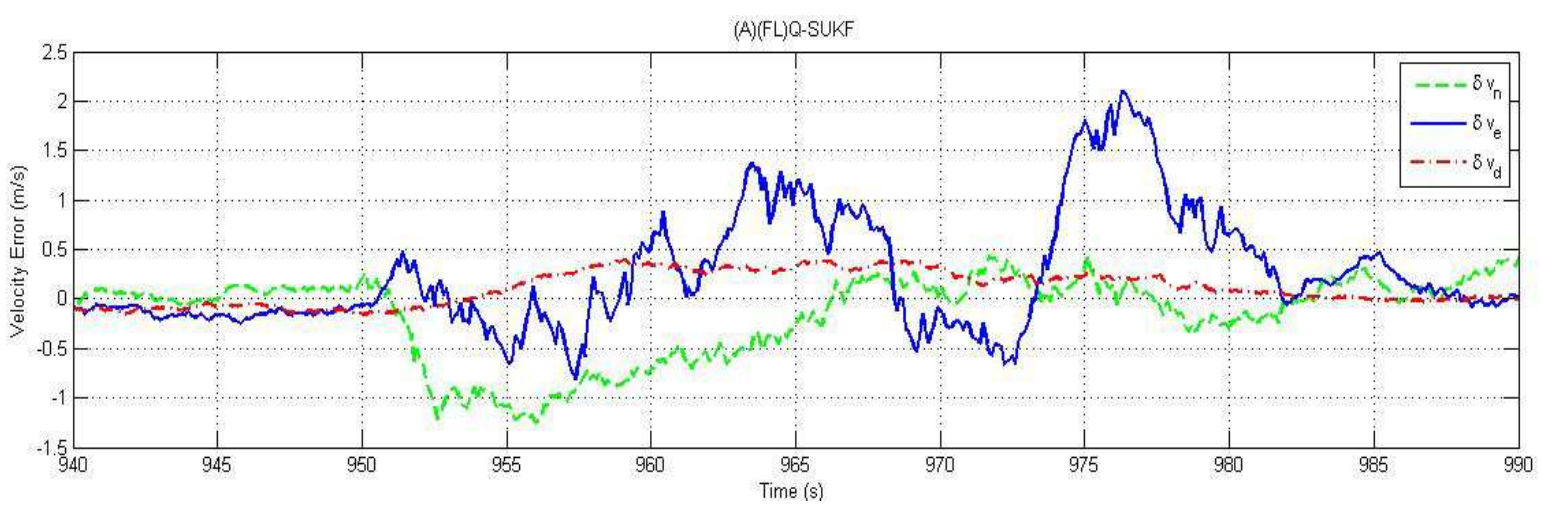

Figure 5b. Velocity error estimated by (A) (FL) Q-SUKF during GPS outage 2

The figures (Figure 6a), (Figure 6b), (Figure 7a) and (Figure $7 \mathrm{~b}$ ) show the position errors during the two periods of GPS outages 1, 2 respectively, before and after the application of the proposed technique of the fuzzy model to Q-SUKF filter.

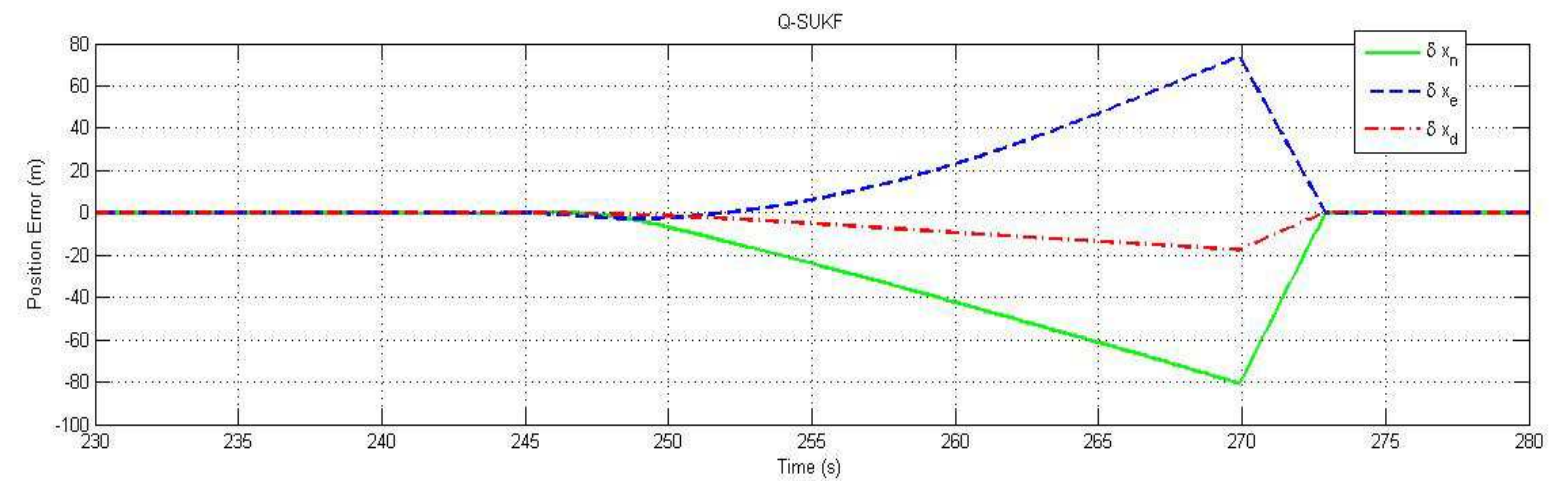

Figure 6a. Position error estimated by Q-SUKF during GPS outage 1 


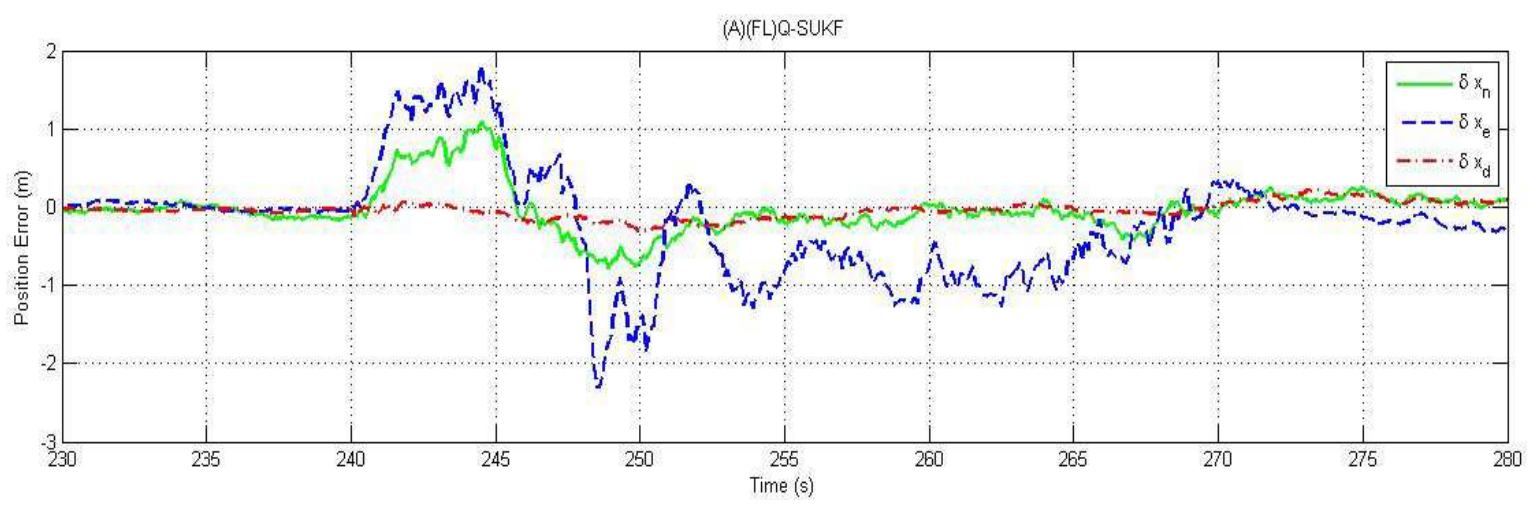

Figure 6b. Position error estimated by (A) (FL) Q-SUKF during GPS outage 1

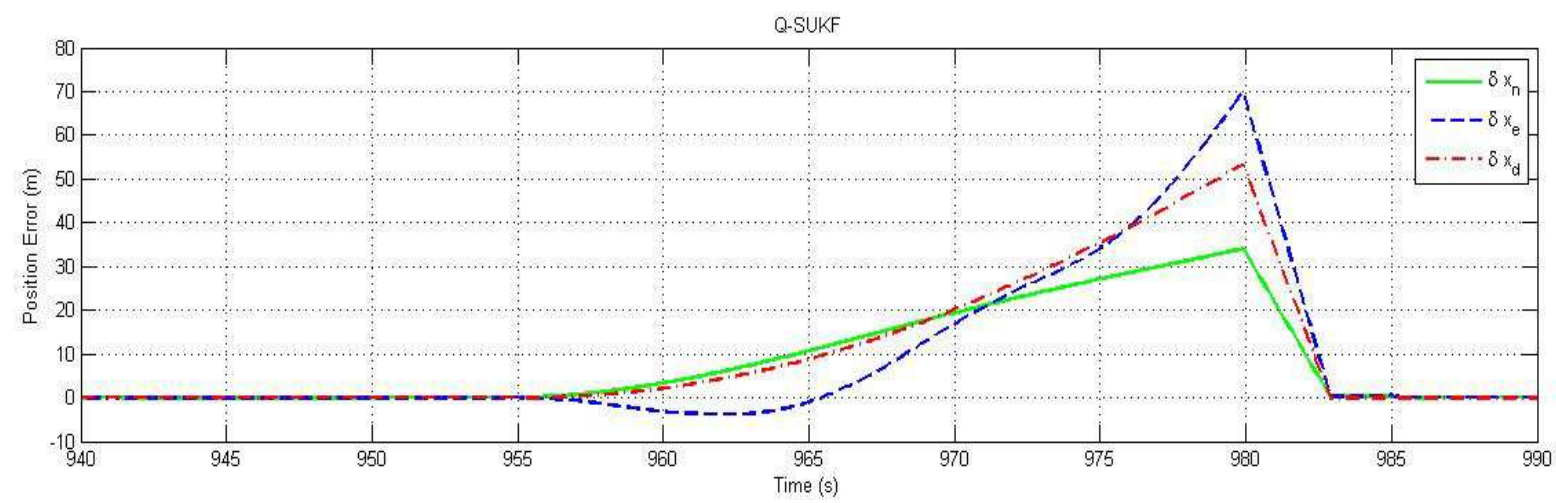

Figure 7a. Position error estimated by Q-SUKF during GPS outage 2

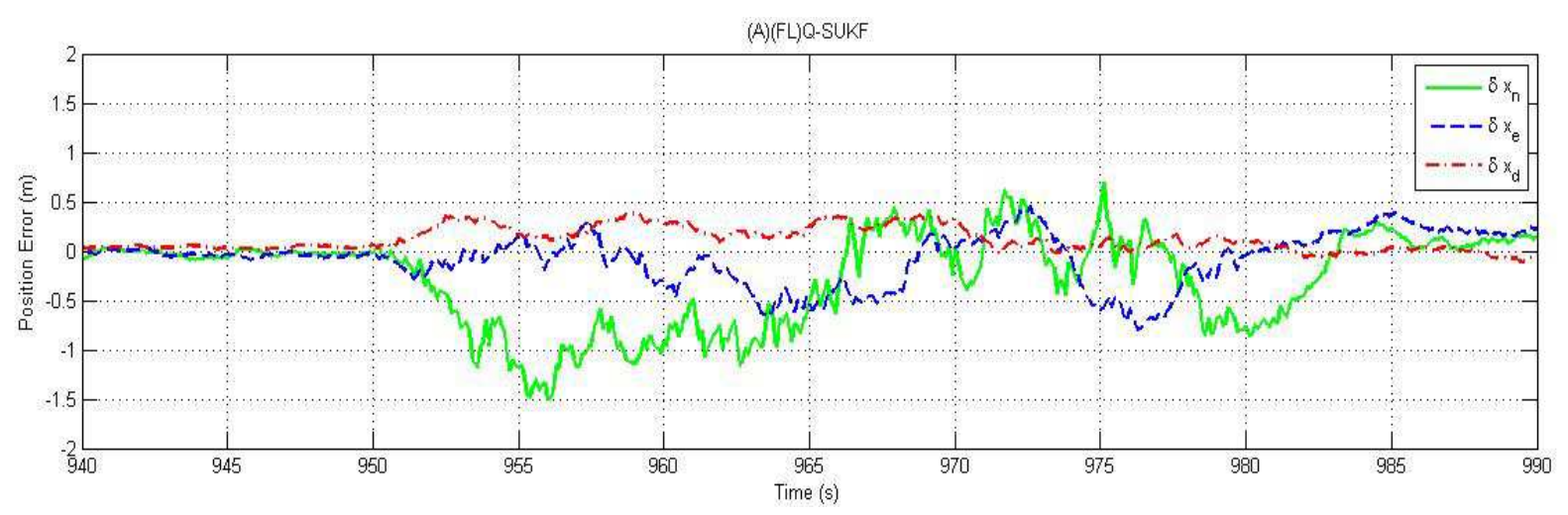

Figure 7b. Position error estimated by (A)(FL) Q-SUKF during GPS outage 2

Table 3. Reduction of the maximum error of the position of Q-SUKF filter after using the fuzzy model

\begin{tabular}{lll}
\hline Maximum Error & Outage GPS 1 & Outage GPS 2 \\
\hline$\delta \mathrm{x}_{\mathrm{n}} \mathrm{Q}-\mathrm{SUKF}$ & $-81,20$ & 34,20 \\
$\delta \mathrm{x}_{\mathrm{n}}(\mathrm{A})(\mathrm{FL}) \mathrm{Q}-\mathrm{SUKF}$ & 1,10 & $-1,50$ \\
Reduction (\%) & $98,64 \%$ & $95,61 \%$ \\
$\delta \mathrm{x}_{\mathrm{e}} \mathrm{Q}-\mathrm{SUKF}$ & & \\
$\delta \mathrm{x}_{\mathrm{e}}(\mathrm{A})(\mathrm{FL}) \mathrm{Q}-\mathrm{SUKF}$ & 74,40 & 70,80 \\
Reduction $(\%)$ & $-2,30$ & $-0,80$ \\
$\delta \mathrm{x}_{\mathrm{d}} \mathrm{Q}-\mathrm{SUKF}$ & $96,90 \%$ & $98,87 \%$ \\
$\delta \mathrm{x}_{\mathrm{d}}(\mathrm{A})(\mathrm{FL}) \mathrm{Q}-\mathrm{SUKF}$ & $-17,60$ & \\
Reduction $(\%)$ & 98,30 & 53,70 \\
\hline
\end{tabular}

We notice in these figures that the maximum errors of the position components $\left(\delta \mathrm{x}_{\mathrm{n}}, \delta \mathrm{x}_{\mathrm{e}}\right.$ et $\left.\delta \mathrm{x}_{\mathrm{d}}\right)$ have been reduced considerably after the application of the proposed technique of the fuzzy model to the Q-SUKF filter during the two periods of GPS outages. The table (Table 3) summarizes the maximum error and the percentage of the reduction of this maximum error for the three position components. The fuzzy model proposed applied to the Q-SUKF filter has provides a significant improvement of $95 \%$ at least in the reduction of the maximum errors on the different components of the position.

In conclusion, although these first results cannot be generalized, they may be promising to give the green light for future research recitals GPS outages in different scenarios of real movement for the interest of a generalization. 


\section{Conclusion}

This paper presents a new hybridization filter of the inertial navigation system with GPS, and magnetometer. This new filter, denoted (A) (FL) Q-SUKF, is based on Q-SUKF filter with the fuzzy modeling. As long as the GPS measurements are reliable, the integration INS/GPS/MAG gives good results. When the measurements of GPS are not reliable or unavailable, the fuzzy model allows the Q-SUKF filter to continue to correct the errors of the estimation of the parameters of navigation (position and velocilty) of the vehicule by providing simulated position and velocity measurements. The results of the experimental validation have shown the effectiveness and the significant impact of the fuzzy technique used with the Q-SUKF filter in the reduction of derivatives of the position and the velocity in case of GPS outages in the tested scenarios. The proposed model has effectively compensated for the absence of GPS update measurements. The (A) (FL) Q-SUKF filter gives us more accurate calculation of the rotation matrix which is integrated in the calculation of the position and velocity, and therefore a significant estimate of the parameters of the navigation system (position and velocity) compared to the QSUKF filter during the absence of GPS signals. The results obtained on synthetic data have shown the contribution of fuzzy logic and have validated the approach proposed.

\section{References}

[1] W. Khoder and B. Jida. A Quaternion Scaled Unscented Kalman Estimator for Inertial Navi- gation States Determination Using INS/GPS/Magnetometer Fusion, Journal of Sensor Technology, vol. 4, no. 2, pp. 101-117, June 2014. http://dx.doi.org/10.4236/jst.2014.42010

[2] R. Babuska, JA Roubos, and H.B. Verbruggen. Identification of MIMO systems by input-output TS fuzzy models, In: The 1998 IEEE international conference on fuzzy systems, vol 1, pp. 657-662, Anchorage, Alaska, 1998.

[3] H. Wang, K. Tanaka and M. Griffin. An approach to fuzzy control of nonlinear systems: stability and design issues, IEEE transactions on fuzzy systems, Vol. 4, pp. 14-23, 1996.
[4] Naderi, M. Aliasghary, A. Pourazar and H. Ghasemzadeh: "A 19MFLIPS CMOS Fuzzy Controller to Control Continuously Variable Transmission Ratio", Ph.D. Research in Microelectronics and Electronics (PRIME), 2011 7th Conference on , vol., no., pp.45,48, 3-7 July 2011.

[5] A. Naderi and S. Ozoguz, "Programmable Implementation of Diamond-Shaped Type-2 Membership Function in CMOS Technology", Journal of Circuit, System and Signal Processing, 2014, doi: 10.1007/s00034-014-9846-x.

[6] J. Abonyi, JA. Roubos, M. Oosterom, and F. Szeifert. Compact TS-Fuzzy Models through Clustering and OLS plus FIS Model Reduction, In : The 10th IEEE international conference on fuzzy systems, vol. 3, pp. 1420-1423, 2001.

[7] J. Dunn. A fuzzy relative to ISODATA process and its use in detecting compact well separated clusters, Journal of Cybernetics, vol.3, n3, pp. 32-57, 1974.

[8] J. Bezdek. Pattern Recognition with Fuzzy Objective Function, Plenum Press, New York, 1981.

[9] V.-H. Grisales Palacio. MODÉLISATION ET COMMANDE FLOUES DE TYPE TAKAGI-SUGENO APPLIQUÉES À UN BIOPROCÉDÉ DE TRAITEMENT DES EAUX USÉES, Ph.D. dissertation, Paul Sabatier University, Toulouse III, 2007.

[10] S.A. Billings, M.J. Korenberg, and S. Chen. Identification of nonlinear output-affine systems using an orthogonal leastsquares algorithm, Int J Sys Sci, vol. 19, pp.1559-1568, 1998.

[11] N. Pal and J. Bezdek. On cluster validity for the fuzzy cmeans model, IEEE Transactions on Fuzzy Systems, vol. no 3, pp. 370-379, 1995.

[12] J. Yu, Q. Cheng and H. Huang. Analysis of the weighting exponent in the FCM, IEEE Transactions on Systems, Man and Cybernetics-Part B: Cybernetics, vol.34, no.1, pp. 634639, 2004.

[13] K. J. Astrom and B. Wittenmark. Computer Controller Systems: Theory and Design, Prentice-Hall, Inc., 1984.

[14] S. E Fahlman. Faster-learning variations on back-propagation: an optimal study, Proceedings of the 1988 Connectionist Models Summer School, pp. 38-51, Carnegic Model University, 1988. 